\title{
A systematic review of nutraceutical interventions for mitochondrial dysfunctions in myalgic encephalomyelitis/chronic fatigue syndrome
}

\author{
Rebekah Maksoud ${ }^{1,2^{*}} \mathbb{1}$, Cassandra Balinas ${ }^{1,2}$, Sean Holden ${ }^{1,2,3}$, Hélène Cabanas ${ }^{1,2}$, Donald Staines ${ }^{1,2}$ \\ and Sonya Marshall-Gradisnik ${ }^{1,2}$
}

\begin{abstract}
Background: Myalgic Encephalomyelitis/Chronic Fatigue Syndrome (ME/CFS) is a debilitating illness, characterised by persistent fatigue that is unrelieved by rest, in combination with a range of other disabling symptoms. There is no diagnostic test nor targeted treatment available for this illness. The pathomechanism also remains unclear. Mitochondrial dysfunctions have been considered a possible underlying pathology based on reported differences including structural and functional changes in ME/CFS patients compared to healthy controls. Due to the potential role that mitochondria may play in ME/CFS, mitochondrial-targeting nutraceutical interventions have been used to potentially assist in improving patient outcomes such as fatigue. The aim of this systematic review is to appraise literature assessing these nutraceuticals as a possible intervention for treating ME/CFS.

Methods: A systematic search of Pubmed, Embase, Medline (EBSCO host) and Web of Science (via Clarivate Analytics) for journal articles published between January 1995 and 10th November 2020 was conducted. Articles assessing nutraceutical interventions and ME/CFS patient outcomes were retrieved. Using specific inclusion and exclusion criteria, the list of articles was further refined. Quality was measured using the Rosendal scale.

Results: Nine intervention studies were included in this review. The studies investigated patient symptom severity changes such as altered fatigue levels in response to mitochondrial-targeting nutraceuticals. Improvements in fatigue levels were observed in six of the nine studies. Secondary outcomes assessed include biochemical, psychological, and quality of life parameters.

Conclusion: There is insufficient evidence on the effectiveness of mitochondria- targeting nutraceuticals in ME/CFS patients. Future well-designed studies are required to elucidate both the involvement of mitochondria in the pathomechanism of ME/CFS and the effect of mitochondrial-modifying agents on illness severity.
\end{abstract}

Keywords: Myalgic encephalomyelitis, Chronic fatigue syndrome, Mitochondria, Nutraceuticals, Intervention

\footnotetext{
*Correspondence: ncned@griffith.edu.au

${ }^{1}$ National Centre for Neuroimmunology and Emerging Diseases (NCNED),

Menzies Health Institute Queensland, Griffith University, Gold Coast, Australia

Full list of author information is available at the end of the article
}

\begin{abstract}
Background
Myalgic Encephalomyelitis/Chronic Fatigue Syndrome (ME/CFS) is a multifaceted illness characterised by persistent fatigue made worse by physical exertion. This fatigue is unrelieved by rest and is accompanied by a combination of symptoms such as immune, cardiovascular, endocrine, and neurological disruption [1-4].
\end{abstract}

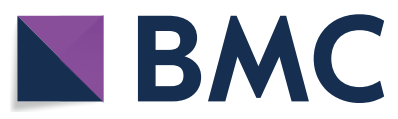

(c) The Author(s) 2021. This article is licensed under a Creative Commons Attribution 4.0 International License, which permits use, sharing, adaptation, distribution and reproduction in any medium or format, as long as you give appropriate credit to the original author(s) and the source, provide a link to the Creative Commons licence, and indicate if changes were made. The images or other third party material in this article are included in the article's Creative Commons licence, unless indicated otherwise in a credit line to the material. If material is not included in the article's Creative Commons licence and your intended use is not permitted by statutory regulation or exceeds the permitted use, you will need to obtain permission directly from the copyright holder. To view a copy of this licence, visit http://creativeco mmons.org/licenses/by/4.0/. The Creative Commons Public Domain Dedication waiver (http://creativecommons.org/publicdomain/ zero/1.0/) applies to the data made available in this article, unless otherwise stated in a credit line to the data. 
Presentation and severity of symptoms varies between patients and leads to decline in overall quality of life, social, occupational, and personal activity where some patients are even bedbound [5]. There is no diagnostic test, thus classification of ME/CFS patients is dependent on case criteria when all other potential clinical explanations are excluded [1-4].

There are four main criteria used both in research and clinical practice to aid diagnosis of ME/CFS: the 1994 Fukuda Criteria (FC), 2003 Canadian Consensus Criteria (CCC), 2011 International Consensus Criteria (ICC), and 2015 Institute of Medicine Criteria (IOMC) [1-4]. FC has been criticised as being too broad, with considerable overlap with other conditions suggesting this is a barrier for appropriate diagnosis, it however, remains the most frequently used case definition [1-4]. The CCC, ICC and IOMC include more ME/CFS specific symptoms such as post- exertional malaise [1, 3, 4]. A common feature in all these definitions is persistent fatigue unrelieved by rest being listed as a cardinal symptom [1-4]. As fatigue is a key diagnostic symptom for ME/CFS, it has been suggested that energy metabolism may be disrupted in ME/ CFS pathomechanisms.

Mitochondria are membrane bound organelles that have diverse physiological roles including energy production and cell cycle control. Mitochondria consist of an inner and outer membrane that separate three core regions: the aqueous regions, the intermembrane space, and the matrix. Within the inner region also resides the electron transport chain (ETC). The ETC comprises of five multi-subunit enzyme complexes (complex I through $\mathrm{V})$ and two electron carriers: coenzyme $\mathrm{Q}_{10}\left(\mathrm{CoQ}_{10}\right)$ and cytochrome $\mathrm{c}$ which are necessary for oxidative phosphorylation leading to production of adenosine triphosphate (ATP)[6]. ATP is the energy currency used by the body to function. In addition to energy production, mitochondria are also key moderators of intracellular calcium $\left(\mathrm{ca}^{2+}\right)$ homeostasis as well as immune regulatory pathways [7, 8]. Due to their physiological versatility, mitochondria have been implicated in numerous pathological conditions including metabolic and age-related disorders as well as ME/CFS [6, 9-27].

A recent systematic review investigated mitochondrial dysfunction in ME/CFS patients compared with healthy controls (HC)[28]. Although disruptions to mitochondrial pathways were documented including changes in mitochondrial respiratory function, metabolites and coenzymes, there was a lack of consistency across the studies, including results and study designs [9-28]. Furthermore, with the low numbers of studies in this field it is difficult to derive appropriate conclusions for mitochondrial dysfunction for the pathomechanism of ME/ CFS [9-28].
Possible mechanisms of mitochondrial involvement in ME/CFS have been described in literature [9-28]. The presentation and severity of ME/CFS symptoms vary from patient to patient [29]. Due to the heterogenous nature of the illness it has been proposed that multiple pathological pathways are implicated in this condition including: muscle function, metabolism, mitochondria, neurological, cardiovascular and immunity [29]. Issues in fatty acid and amino acid metabolism as well as inefficient ATP synthesis have been suggested to have developed in ME/CFS patients due to dysregulation of mitochondrial or metabolic pathways [29].

ME/CFS patients have used a variety of different mitochondrial- based nutraceuticals including nicotinamide adenine dinucleotide hydrogen (NADH), coenzyme $\mathrm{Q}_{10}$ $\left(\mathrm{CoQ}_{10}\right)$ and Acetyl L-Carnitine (ALC) as part of their treatment regime [2, 30-32]. Patients use these treatments either in isolation or in combination with a cocktail of other nutraceutical and/ or pharmaceutical- based products in the attempt to ameliorate their symptoms. A systematic appraisal of these reports and the impact they have on mitochondrial pathways affected in ME/CFS has not been conducted.

Using our previous publication as a basis we have expanded the search to assess the effectiveness of nutraceutical interventions in treating ME/CFS symptoms by measuring patient-centred outcomes such as fatigue levels [28].

\section{Methods}

This systematic review followed Preferred Reporting Items for Systematic Reviews and Meta-Analyses (PRISMA) and Cochrane review guidelines (Fig. 1). Systematic searches of the databases Pubmed, Embase, Medline (EBSCO host) and Web of Science (via Clarivate Analytics) were conducted to collect relevant literature. Independent literature searches were conducted by RM and CB between January 1995 to 10th November 2020. No additional papers were found following reference list checking and citation searching. All potentially relevant papers have been included in this review (Additional file 1).

\section{Inclusion and exclusion criteria}

Studies were selected if they contained at least one mitochondrial or intervention search term AND at least one ME/CFS search term (Additional file 1) as well as satisfying the following inclusion criteria: (i) interventional study published after 1994 (ii) study conducted on human participants aged 18 years or older; (iii) full-text available in English (iv) reporting of original research only; (v) diagnosis of ME/CFS was made using: FC (1994), CCC 


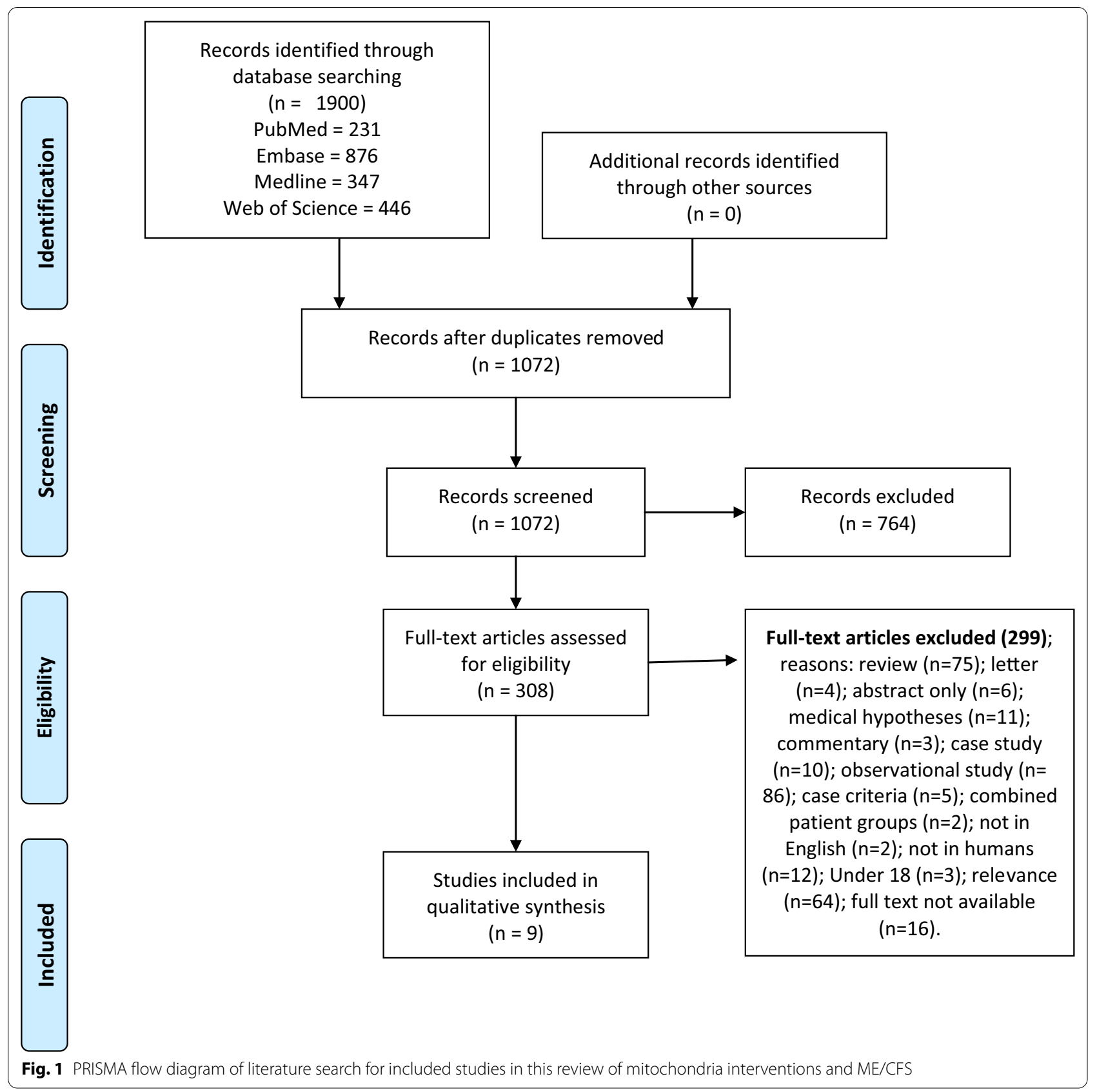

(2003), ICC (2011) or IOMC (2015) only; (vi) studies using nutraceuticals to target mitochondrial disruption in $\mathrm{ME} / \mathrm{CFS}$.

Studies were excluded from the review if they did not contain at least one mitochondrial or intervention search term AND at least one ME/CFS search term in the title or abstract. Articles were also excluded if they met any of the following criteria: (i) was an observational study (ii) written prior to 1995 , as the study defining FC was published in December 15th 1994; (iii) not available as full- text; (iv) not published in English; (v) was a nonoriginal study type including: reviews, duplicate studies or case reports; (vi) use of diagnostic criteria other than FC, CCC, ICC or IOMC; (vii) studies not within the scope of this review (Additional file 2).

\section{Selection of studies}

All articles that were retrieved from each database were stored in the reference management software package Endnote X9.2. Duplicates were automatically removed 
and then the result was manually checked to confirm the removal of all duplicates. Titles and abstracts were screened for the listed key words. Articles that contained one mitochondrial or intervention term and one ME/ CFS term were retained. Articles that did not contain the aforementioned key words were omitted. The remaining articles were assessed for suitability against inclusion and exclusion criteria and those that met eligibility criteria were selected. The described processes were conducted independently by authors RM and CB. The outcome of this screening process was discussed and deemed correct by both authors. The included articles were reassessed by all other listed authors for appropriateness.

\section{Data extraction}

Relevant data were extracted from each of the studies (Table 1), this includes: (i) study design; (ii) treatment; (iii) reported adverse effects; (iv) treatment duration; (v) ME/CFS case definition; (vi) country; (vii) sample size; (viii) age of participants; (ix) sex, percentage of female participants; $(x)$ illness duration; (xi) body mass index; (xii) weight; (xiii) duration of treatment; (xiv) washout period; (xv) treatment intervention; (xvi) control intervention; (xvii) fatigue outcome; (xviii) fatigue result.

\section{Quality assessment}

Quality and bias were assessed using the Rosendal scale [33]. This scale combines the PEDro scale [34], Jadad scoring system [35] and Delphi list [36]. This Rosendal scale was selected as the PEDro scale, Jadad scoring system and Delphi list have been extensively evaluated and validated. A Rosendal score of $60 \%$ is considered as excellent methodological quality [33]. Item 16 was excluded due to exercise performance outcomes not being measured in this review and item 15 was replaced with a checklist item that addresses a washout period in crossover studies as conducted in Campagnolo et al.'s study [5]. No studies were excluded based on quality assessment results. Quality assessment was individually conducted by RM and CB.

\section{Results}

A total of 1900 papers were retrieved from PubMed (231), Embase (876), Medline (347) and Web of Science (446). Duplicates were then removed, and inclusion and exclusion criteria were applied to the remaining articles. From this process the total number of articles was refined to nine. This selection process conducted according to PRISMA guidelines is summarised in Fig. 1.

\section{Overview of papers}

Study characteristics are presented in Table 1. All included papers are intervention based. One study comprised of two experiments the initial experiment was an open-labelled pilot trial (OPT) and the subsequent experiment was a randomised control trial (RCT) with parallel design [37]. Four of the other included studies were RCT where two were crossover design and two were a parallel design [30,31, 38, 39]. Two of the studies were OPT $[40,41]$. One of the studies was a randomised control pilot trial [32]. The final study was a proof of concept study [42].

\section{Participant and study characteristics}

Participant characteristics are also presented in Table 1. All nine papers used the FC to diagnose ME/CFS [30$32,37-42]$. The average sample size across the studies was 28.7 for treatment groups and 37.5 for the placebo control groups. The average age was 40.12 for treatment groups and 43.63 for the placebo control group. Females made up a greater proportion of the participants where $75.2 \%$ of the participants in the treatment group and $84 \%$ of the participants in the placebo control group were female. The average illness duration was 9.7 years for treatment groups and 13.7 years for the placebo control groups. All studies except one reported fatigue as a primary outcome [31, 32, 37-42]. To measure fatigue a variety of different resources were used including the: Fatigue Index Symptom Questionnaire (Fis-40), 50 item questionnaire based on the FC, Chalder Fatigue Score, visual analogue scale (VAS) for Fatigue and Multidimensional Fatigue Inventory (MFI) [31, 32, 37-42].

\section{Interventions on primary outcomes}

Fatigue was the primary outcome measure in eight out of nine studies [31, 32, 37-42]. From these studies, six found significant differences in fatigue levels following an intervention [31, 32, 39-42]. When receiving a combination treatment of $\mathrm{CoQ}_{10}$ and $\mathrm{NADH}$ a significant reduction in Fis-40 scores were reported in one study [31]. Additionally, consumption of NADH alone, also resulted in significantly reduced fatigue scores compared to the placebo control group [32]. Combination treatments including: KPAX002 (methylphenidate hydrochloride accompanied by a mitochondrial modulator) and another range of mitochondrial targeting nutrients significantly reduced symptom scores [39, 41, 42]. One KPAX002 study found that the most significant response was with those who had higher symptom severity. ALC and propionyl-L-carnitine (PLC) significantly reduced mental fatigue and general fatigue, respectively. However, combination treatments of ALC AND PLC treatments was a less effective intervention [40]. Two studies reported no significant changes in fatigue levels following intervention $[37,38]$. One study's primary endpoint was to investigate the effect of $\mathrm{CoQ}_{10}$ plus $\mathrm{NADH}$ supplementation 


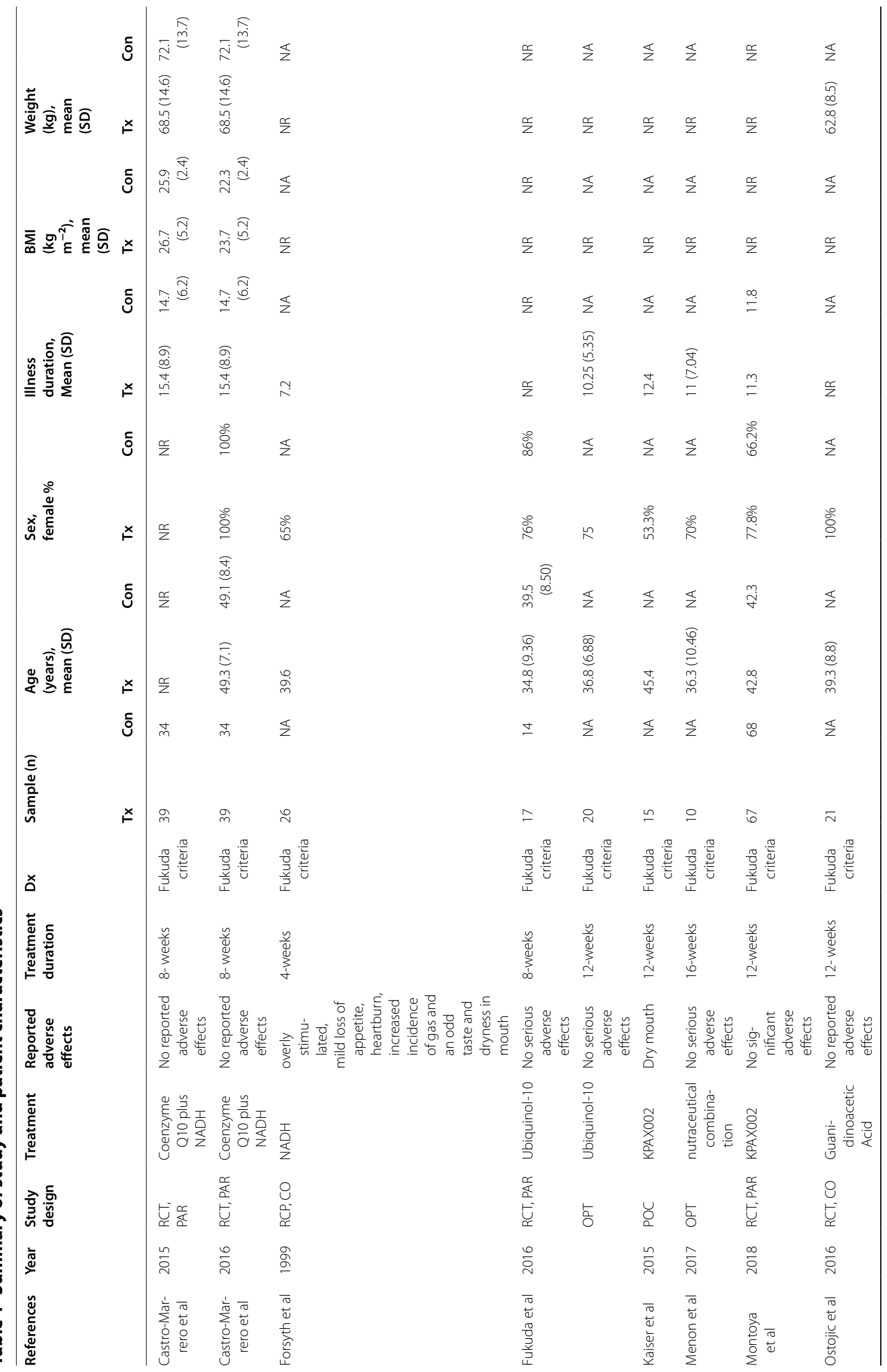




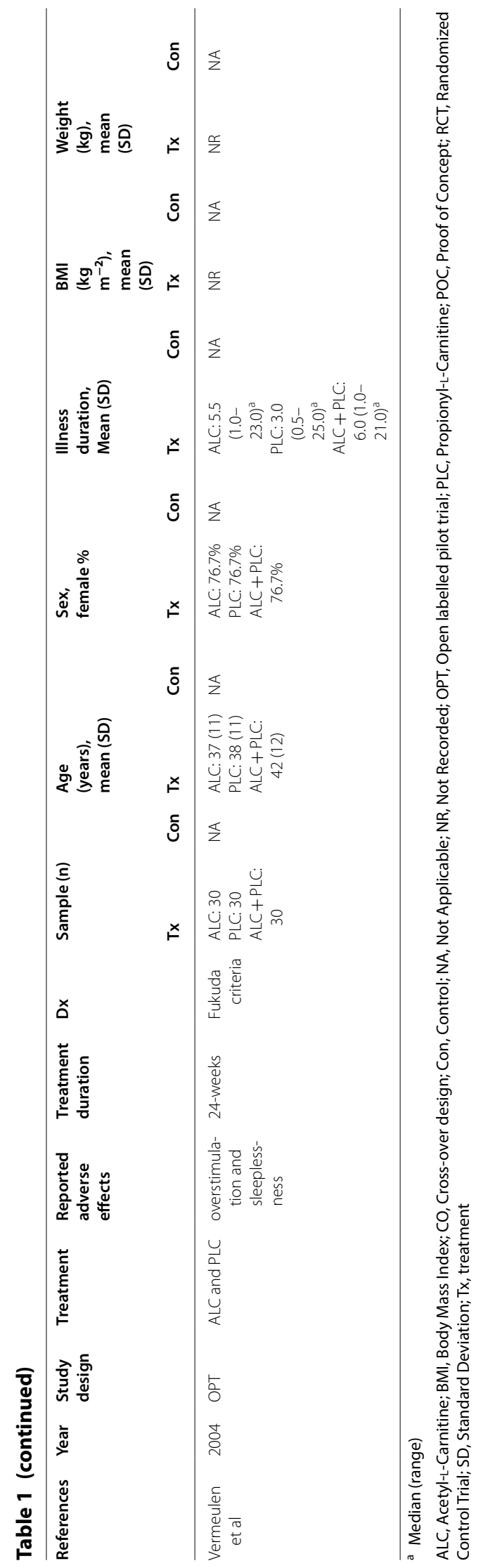


on age-predicted maximum heart rate when completing a cycle ergometer task [30]. Participants in the intervention group reported significantly lower max HR. Perception of fatigue in this group was significantly reduced in all follow up visits in the intervention group compared with the placebo group [30].

\section{Interventions on secondary outcomes}

Multiple secondary outcomes were investigated including physical or biochemical parameters, psychological, quality of life and pain scores. One study reported significantly higher nicotinamide adenine dinucleotide $\left(\mathrm{NAD}^{+} / \mathrm{NADH}\right), \mathrm{CoQ}_{10}$, adenosine triphosphate (ATP) and citrate synthase levels as well as a significant reduction in lipoperoxides in ME/CFS patients using $\mathrm{CoQ}_{10}$ and NADH [31]. Another study found that patients using guanidinoacetic acid (GAA) had significantly higher muscular creatine levels as well as improved muscular strength and aerobic power [38]. In a study that investigated depression scores following intervention with ubiquinol-10, scores were found to be dependent on an increase in total plasma $C_{0} Q_{10}$ levels [37]. A study that investigated the effect of $\mathrm{CoQ}_{10}$ and $\mathrm{NADH}$ on max heart rate when completing a cycle ergometer task found no significant difference in sleep and pain scores, however, fatigue scores were significantly lower [30]. Vermeulen et al. investigated the effects of ALC, PLC and a combinatorial treatment of ALC and PLC [40]. Performance on the Stroop attention concentration test was significantly improved in all groups while pain scores were not significantly different between either of the groups [40].

\section{Quality assessment}

Quality assessment scores for each study can be found in Additional file 2. Seven of the studies in this review were determined high quality (Rosendal score $>60 \%$ ) [30-32, 37-40]. The most effectively addressed items were number one, six and ten. These items assess whether the study had a clear description of the inclusion and exclusion criteria, incorporation of baseline variables and whether details of participants who did not complete the study course were provided, respectively. The least addressed item was number nine which assesses the method of blinding participants and whether the effectiveness of blinding was described (Table 2).

\section{Discussion}

The use of nutritional supplements has been reported by ME/CFS patients for management of their condition [3032, 37-42]. This systematic review aims to investigate the possible effect of mitochondrial-modifying nutraceuticals on ME/CFS patient outcomes such as fatigue and quality of life, sleep, and cognitive ability.
Mitochondrial disruption in ME/CFS patients was investigated previously in a systematic review conducted by Holden et al.[28] that reported inconsistent evidence linking mitochondrial disruption and ME/CFS [16, 19, $21,26,27]$. No significant differences in mitochondrial deoxyribonucleic acid were found, strongly suggesting that ME/CFS is not a primary mitochondrial disorder [24]. The publication by Holden et al. only included observational studies [9-28]. This present interventional systematic review was conducted to complement Holden et al's study by investigating whether mitochondrial disruption has a role in ME/CFS pathomechanism as well as patient outcomes, when treated with mitochondrialtargeting nutraceuticals. This manuscript is the first to investigate this topic.

Key terms were derived from Nicolson et al.s review which featured an extensive list of nutraceutical agents used to treat mitochondrial dysfunction [43]. Limitations on relying on this developed list is exclusion of potentially relevant articles based on keywords selected. The final list of included articles has been reviewed and all appropriate articles have been incorporated in the present review. Some studies that investigated mitochondrial-targeting nutraceuticals that were on Nicolson's list were not included in the final review due to not meeting criteria, for example, Comhaire et al. [44]. The diagnostic criteria used in this study is unclear therefore the manuscript was not selected [44]. This study investigated use of the nutraceutical, sodium dichloroacetate for treatment of ME/CFS [44].

Majority of the participants were female $(75.2 \%$ for treatment group and $84 \%$ for placebo controls) with an average age of 40.12 years for the treatment group and 43.63 for controls. This is consistent with literature stating that ME/CFS is mostly reported in females aging between 35 and 45 years $[19,45]$. Age and sex-matching participants is essential in mitochondrial studies due to age-related mitochondrial decline and sex-associated differences, including uncoupled respiration, citrate synthase activity and ATP levels [46]. One study specifically recruited only females to increase sample homogeneity [38]. Only two studies reported data on race or ethnicity where most of the participants were Caucasian [32, 39]. Lack of inclusion of minority groups, however, will result in a sample that doesn't appropriately represent the population [47].

Information on diet restrictions was provided in two studies [38, 39]. Due to possible food-drug and food-food interactions, consumption of certain foods may influence response to treatment, hence, a diet record is a necessary consideration for future studies [48].

All the studies included in this review have utilised the FC to diagnose ME/CFS patients [2]. While the FC 
Table 2 Summary of primary/secondary outcome results

\begin{tabular}{|c|c|c|}
\hline References (date) & Primary/ Secondary outcome measures (s) & Results \\
\hline Castro Marrero et al. (2015) & $\begin{array}{l}\text { NAD }+/ \mathrm{NADH} \\
\text { CoQ10 } \\
\text { ATP } \\
\text { Citrate Synthase } \\
\text { Lipoperoxides }\end{array}$ & $\begin{array}{l}\text { NAD + } / \text { NADH: significantly higher }(p<0.001) \\
\text { CoQ }_{10} \text { : significantly higher }(p<0.05) \\
\text { ATP: significantly higher }(p<0.05) \\
\text { Citrate Synthase: significantly higher }(p<0.05) \\
\text { Lipoperoxides: significantly lower }(p<0.05)\end{array}$ \\
\hline Castro Marrero et al. (2016) & FIS-40 & $\begin{array}{l}\text { Sleep: NS } \\
\text { Pain: NS } \\
\text { Fatigue: significantly lower }(p=0.03)\end{array}$ \\
\hline Forsyth et al. (1999) & NA & NA \\
\hline Fukuda et al. (2016) & CES-D & $\begin{array}{l}\text { NS. Increase in depressive symptom scores were } \\
\text { dependent on increase in total plasma } \operatorname{CoQ}_{10} \text { levels }\end{array}$ \\
\hline Kaiser et al. (2015) & NA & NA \\
\hline Menon et al. (2017) & $\begin{array}{l}\text { Clinical Global Impression Scale (CGI) } \\
\text { Patient Global Impression Measures (PGI) } \\
\text { Insomnia Severity Index (ISI) }\end{array}$ & $\begin{array}{l}\text { CGI: Significantly improved }(p=0.014) \\
\text { PGI: NS } \\
\text { ISI: Significantly improved }(p=0.017)\end{array}$ \\
\hline Montoya et al. (2018) & $\begin{array}{l}\text { VAS for fatigue } \\
\text { Concentration disturbance symptoms }\end{array}$ & $\begin{array}{l}\text { VAS for fatigue: NS } \\
\text { Concentration disturbance symptoms: NS }\end{array}$ \\
\hline Ostojic et al. (2016) & $\begin{array}{l}\text { Muscular Creatine Levels } \\
\text { Muscular Strength and Aerobic Power }\end{array}$ & $\begin{array}{l}\text { Muscular creatine levels: Significantly higher }(p<0.01) \\
\text { Muscular Strength and Aerobic Power: }(p<0.05)\end{array}$ \\
\hline Vermeulen et al. (2004) & $\begin{array}{l}\text { Stroop attention concentration test } \\
\text { McGill Pain Questionnaire }\end{array}$ & $\begin{array}{l}\text { Stroop attention concentration test } \\
\text { ALC: } p<0.001 \\
\text { PLC: } p=0.011 \\
\text { ALC+PLC: } p=0.004 \\
\text { MCGill Pain Questionnaire } \\
\text { ALC: NS } \\
\text { PLC: } p=N S \\
\text { ALC }+ \text { PLC: NS }\end{array}$ \\
\hline
\end{tabular}

ALC, Acetyl-L-Carnitine; ATP, Adenosine Triphosphate; CES-D, Center for Epidemiological Studies Depression CGI, Clinical Global Impression Scale; CoQ10, Coenzyme Q10; FIS-40, Fatigue Impact Scale; ISI, Insomnia Severity Index; NAD, Nicotinamide Adenine Dinucleotide; NADH, Nicotinamide Adenine Dinucleotide Hydrogen; NA, Not Applicable; PGI, Patient Global Impression Measures; PLC, Propionyl-L-Carnitine; NS, Non-significant; VAS, Visual Analogue Scale

is the most widely utilised, is considered too broad and represents a more heterogenous set of patients with overlap with other illnesses such as depression [3]. Use of the more refined definitions including the CCC, ICC and IOMC may improve the overall sensitivity of these experiments; which is an important consideration for future studies $[1,3,4]$.

Across the studies, an extensive range of different study types were identified including pilot trials and RCT. All cross-over studies effectively implemented a washout period [32, 38]. Some studies lacked an established placebo group [41, 42]. The studies that did have a placebo group indicated that there was a noticeable effect of the placebo [32]. Implementation of a placebo group is important to account for the placebo effect contribution to the observed results especially where subjective measures are concerned. Overall improvement in study design in future studies is paramount including repeating of open pilot studies as a RCT.

A range of different criteria to measure fatigue were used across the studies including: chalder fatigue score, Fis-40, visual analogue scale for fatigue and MFI. Due to this variety, it is difficult to adequately compare each study. One study used their own developed criteria based on the FC [32]. Reproducibility was tested for this criteria; however, this impact was only observed in one study and lacks validation [32]. Therefore, use of standardised, validated tools as conducted by all the other included studies is recommended for future research. Fatigue was calculated based on self-report questionnaires, these reports are subject to bias including selective recall and social desirability [49].

Tolerance is an important consideration for long-term use of natural supplements. Tolerance was assessed in eight of the studies [30-32, 37-41]. Across the studies adverse effects that were described include over-stimulation, heartburn, dry mouth and mild loss of appetite [30-32, 37-41]. Due to concomitant medications not being controlled in the study, understanding the adverse effects caused by the nutraceuticals alone is hindered [41]. Majority of the studies investigated short-term side effects whereas long-term effects of supplement use for ME/CFS patients has not been determined. Replication of these studies for longer durations to assess long-term implications of use is necessary. 
One possible outcome highlighted in our previous mitochondrial paper is that disruption of mitochondrial pathways may be one portion of a much more complex, multifactorial pathological process involving multiple pathways such as $\mathrm{Ca}^{2+}$ signalling $[18,19]$. Some mitochondrial processes are $\mathrm{Ca}^{2+}$-dependent [7]. Additionally, mitochondria have a role in $\mathrm{Ca}^{2+}$-homeostasis [50]. Importantly, impairments in $\mathrm{Ca}^{2+}$-dependent pathways results in oxidative stress that have been reported in ME/ CFS patients [32, 39-42].

A review by Wood et al. investigated mitochondrial involvement in ME/CFS, oxidative stress and therapeutic potential of antioxidant therapies including $\mathrm{CoQ}_{10}$ in ME/CFS patients [51]. There is still insufficient evidence of mitochondrial involvement in ME/CFS and further research is required to establish appropriate grounds for treatments [28].

Currently there is limited evidence available on the efficacy of mitochondria interventions for treatment of ME/CFS symptoms. Additional well-designed studies are required to understand the impact of these nutraceuticals on ME/CFS patients. Furthermore, in a letter to the editor written by Ostojic et al. the role of creatine in treatment of ME/CFS was discussed. The overall message of the excerpt was to refrain from use until better evidence is available including: dosing, treatment duration and side-effects [52]. This suggestion can be applied to all other treatments assessed in this systematic review [52].

\section{Quality assessment}

A Rosendal score of $60 \%$ is considered as excellent methodological quality [33]. This score was achieved by seven of the included studies [30-32, 37-40]. All papers were effective at describing eligibility and exclusionary criteria. Majority of the studies received lower scores due to lack of randomisation and blinding of participants and assessors. The least addressed item was number nine. Item nine assesses the method of blinding and whether the successfulness of blinding has been discussed. These details were only provided by one study. Important considerations for future studies include selection and justification of an appropriate sample size, incorporation of randomisation and blinding processes and ensuring that evaluation of blinding measures have been discussed. Additionally, appropriate statistical measures must be used; this includes, selection of statistical tests based on normality assessment as well as adjusting for multiple parameters. Sample size must also be considered statistically sufficient.

\section{Conclusion}

The aim of this systematic review was to evaluate available research on mitochondrial-based nutraceutical interventions. Due to numerous limitations associated with these studies such as low availability of studies investigating this topic, lack of consistency of measuring tools for fatigue used, low sample size and short study duration, it is difficult to make conclusions on whether these interventions have an effect on mitochondrial function in ME/CFS patients. Therefore, future well-designed studies are required to determine mitochondrial involvement in ME/CFS pathology and the possible effectiveness of these interventions in treating ME/CFS. These findings may help direct future research in this field.

\section{Supplementary Information}

The online version contains supplementary material available at https://doi. org/10.1186/s12967-021-02742-4.

Additional file 1. Raw search code.

Additional file 2. Quality assessment summary and Rosendal score of studies KB).

\section{Abbreviations}

ALC: Acetyl-L-Carnitine; ATP: Adenosine triphosphate; Ca2 + : Calcium; CCC : Canadian Consensus Criteria; $\mathrm{COQ}_{10}$ : Coenzyme $\mathrm{Q}_{10}$; ETC: Electron Transport Chain; HC: Healthy control; FIS: Fatigue Index Symptom; FC: Fukuda Criteria; GAA: Guanidinoacetic acid; ICC: International Consensus Criteria; IOMC: Institute of Medicine Criteria; MeSH: Medical subject headings; mRNA: Messenger Ribonucleic Acid; mtDNA: Mitochondrial DNA; MFI: Multidimensional Fatigue Inventory ME/CFS: Myalgic Encephalomyelitis/ Chronic Fatigue Syndrome; NK: Natural Killer; NAD: Nicotinamide adenine dinucleotide; NADH: Nicotinamide adenine dinucleotide hydrogen; OPT: Open-labelled pilot trial; PRISMA: Preferred Reporting Items for Systematic Reviews; PLC: Propionyl-L-Carnitine; RCT: Randomised control trials; VAS: Visual Analogue Scale.

\section{Acknowledgements \\ Not applicable.}

\section{Authors' contributions}

DS and SMG developed the concept for this systematic review. RM and SH designed the search strategy for this review based on our previous manuscript (https://doi.org/10.1186/s12967-020-02452-3). RM performed the primary literature search, screening of papers, analysis of results and primary quality assessment. CB conducted the secondary publication search, quality assessment and critically reviewed the drafts of this manuscript. All authors read and approved the final manuscript.

\section{Funding}

This research was supported by the Stafford Fox Medical Research Foundation, the Mason Foundation, Mr. Douglas Stutt, Blake Beckett Foundation, Alison Hunter Memorial Foundation, the McCusker Charitable Foundation, Buxton Foundation, Mr and Mrs Stewart, Henty Community, Henty Lions Club and the Change for ME Charity. The funders had no role in study design, data collection and analysis, decision to publish, or preparation of the manuscript.

Availability of data and materials

All data generated or analysed during this study are included in this published article.

Ethics approval and consent to participate

Not applicable. 


\section{Consent for publication \\ Not applicable.}

\section{Competing interests}

The authors declare no conflicts of interest. The lead author confirms that this manuscript is an accurate, honest and transparent account of the study undertaken and reported, with no aspects being omitted and any discrepancies explained.

\section{Author details \\ ${ }^{1}$ National Centre for Neuroimmunology and Emerging Diseases (NCNED), \\ ${ }^{2}$ Consortium Health International for Myalgic Encephalomyelitis, Griffith Coast, Australia. \\ Received: 10 December 2020 Accepted: 5 February 2021 Published online: 17 February 2021} Menzies Health Institute Queensland, Griffith University, Gold Coast, Australia. University, Gold Coast, Australia. ${ }^{3}$ School of Medicine, Griffith University, Gold

\section{References}

1. Carruthers BM, Van de Sande MI, De Meirleir KL, Klimas NG, Broderick G, Mitchell T, et al. Myalgic encephalomyelitis: international consensus criteria. J Intern Med. 2011;270(4):327-38.

2. Fukuda K, Straus SE, Hickie I, Sharpe MC, Dobbins JG, Komaroff A. The chronic fatigue syndrome: a comprehensive approach to its definition and study. International Chronic Fatigue Syndrome Study Group. Ann Intern Med. 1994;121(12):953-9.

3. Committee on the Diagnostic Criteria for Myalgic Encephalomyelitis/ Chronic Fatigue Syndrome, Board on the Health of Select Populations, Institute of Medicine. Beyond Myalgic Encephalomyelitis/Chronic Fatigue Syndrome: Redefining an Illness [Internet]. Washington (DC): National Academies Press (US); 2015 [cited 2020 Mar 25]. (The National Academies Collection: Reports funded by National Institutes of Health). Available from: http://www.ncbi.nlm.nih.gov/books/NBK274235/.

4. Carruthers BM, Jain AK, Meirleir KLD, Peterson DL, Klimas NG, Lerner AM, et al. Myalgic encephalomyelitis/chronic fatigue syndrome. J Chronic Fatigue Syndr. 2003;11(1):7-115

5. Campagnolo N, Johnston S, Collatz A, Staines D, Marshall-Gradisnik S. Dietary and nutrition interventions for the therapeutic treatment of chronic fatigue syndrome/myalgic encephalomyelitis: a systematic review. J Hum Nutr Diet. 2017;30(3):247-59.

6. Osellame LD, Blacker TS, Duchen MR. Cellular and molecular mechanisms of mitochondrial function. Best Pract Res Clin Endocrinol Metab. 2012;26(6):711-23.

7. Pivovarova NB, Andrews SB. Calcium-dependent mitochondrial function and dysfunction in neurons. FEBS J. 2010;277(18):3622-36.

8. Angajala A, Lim S, Phillips JB, Kim J-H, Yates C, You Z, et al. Diverse roles of mitochondria in immune responses: novel insights into immuno-metabolism. Front Immunol. 2018:9:1.

9. Armstrong CW, McGregor NR, Lewis DP, Butt HL, Gooley PR. Metabolic profiling reveals anomalous energy metabolism and oxidative stress pathways in chronic fatigue syndrome patients. Metabolomics. 2015;11(6):1626-39.

10. Billing-Ross P, Germain A, Ye K, Keinan A, Gu Z, Hanson MR. Mitochondrial DNA variants correlate with symptoms in myalgic encephalomyelitis/ chronic fatigue syndrome. J Transl Med. 2016;14:1.

11. Booth NE, Myhill S, McLaren-Howard J. Mitochondrial dysfunction and the pathophysiology of myalgic encephalomyelitis/chronic fatigue syndrome (ME/CFS). Int J Clin Exp Med. 2012;5(3):208-20.

12. Castro-Marrero J, Cordero MD, Sáez-Francas N, Jimenez-Gutierrez C, Aguilar-Montilla FJ, Aliste L, et al. Could mitochondrial dysfunction be a differentiating marker between chronic fatigue syndrome and fibromyalgia? Antioxid Redox Signal. 2013;19(15):1855-60.

13. Germain A, Ruppert $D$, Levine $S M$, Hanson MR. Metabolic profiling of a myalgic encephalomyelitis/chronic fatigue syndrome discovery cohort reveals disturbances in fatty acid and lipid metabolism. Mol BioSyst. 2017;13(2):371-9.

14. Light KC, Agarwal N, lacob E, White AT, Kinney AY, VanHaitsma TA, et al. Differing leukocyte gene expression profiles associated with fatigue in patients with prostate cancer versus chronic fatigue syndrome. Psychoneuroendocrinology. 2013;38(12):2983-95.

15. Maes M, Mihaylova I, Kubera M, Uytterhoeven M, Vrydags N, Bosmans E. Coenzyme Q10 deficiency in myalgic encephalomyelitis / chronic fatigue syndrome (ME/CFS) is related to fatigue, autonomic and neurocognitive symptoms and is another risk factor explaining the early mortality in ME/CFS due to cardiovascular disorder. Neuroendocrinol Lett. 2009;30(4):470-6.

16. Mandarano A.H., Maya J., Giloteaux L., Peterson D.L., Maynard M., Gottschalk C.G., et al. Myalgic encephalomyelitis/chronic fatigue syndrome patients exhibit altered T cell metabolism and cytokine associations. J Clin Invest. 2019;((Mandarano A.H.; Maya J.; Giloteaux L.; Peterson D.L.; Maynard M.; Gottschalk C.G.; Hanson M.R.)).

17. Naviaux RK, Naviaux JC, Li K, Bright AT, Alaynick WA, Wang L, et al. Metabolic features of chronic fatigue syndrome. Proc Natl Acad Sci USA. 2016:113(37):E5472-80

18. Nguyen T, Staines D, Nilius B, Smith P, Marshall-Gradisnik S. Novel identification and characterisation of Transient receptor potential melastatin 3 ion channels on Natural Killer cells and B lymphocytes: Effects on cell signalling in Chronic fatigue syndrome/Myalgic encephalomyelitis patients. Biol Res. 2016;49:1.

19. Nguyen T, Staines D, Johnston S, Marshall-Gradisnik S. Reduced glycolytic reserve in isolated natural killer cells from myalgic encephalomyelitis/ chronic fatigue syndrome patients: a preliminary investigation. Asian Pac J Allergy Immunol. 2019;37(2):102-8.

20. Plioplys AV, Plioplys S. Electron-microscopic investigation of muscle mitochondria in chronic fatigue syndrome. Neuropsychobiology. 1995;32(4):175-81.

21. Shungu DC, Weiduschat N, Murrough JW, Mao X, Pillemer S, Dyke JP, et al. Increased ventricular lactate in chronic fatigue syndrome. III. Relationships to cortical glutathione and clinical symptoms implicate oxidative stress in disorder pathophysiology. NMR Biomed. 2012;25(9):1073-87.

22. Sweetman E, Ryan M, Edgar C, Mackay A, Vallings R, Tate W. Changes in the transcriptome of circulating immune cells of a New Zealand cohort with myalgic encephalomyelitis/chronic fatigue syndrome. Int J Immunopathol Pharmacol. 2019;33:1.

23. Tomas C, Brown A, Strassheim V, Elson J, Newton J, Manning P. Cellular bioenergetics is impaired in patients with chronic fatigue syndrome. PLoS ONE. 2017; 12:10.

24. Venter M, Tomas C, Pienaar IS, Strassheim V, Erasmus E, Ng W-F, et al. MtDNA population variation in Myalgic encephalomyelitis/Chronic fatigue syndrome in two populations: a study of mildly deleterious variants. Sci Rep. 2019;9:1.

25. Yamano E, Sugimoto M, Hirayama A, Kume S, Yamato M, Jin G, et al. Index markers of chronic fatigue syndrome with dysfunction of TCA and urea cycles. Sci Rep. 2016;6:1.

26. Missailidis D, Annesley SJ, Allan CY, Sanislav O, Lidbury BA, Lewis DP, et al. An Isolated Complex V Inefficiency and Dysregulated Mitochondrial Function in Immortalized Lymphocytes from ME/CFS Patients. Int J Mol Sci. 2020;21:3.

27. Missailidis D, Sanislav O, Allan CY, Annesley SJ, Fisher PR. Cell-based blood biomarkers for myalgic encephalomyelitis/chronic fatigue syndrome. Int J Mol Sci. 2020;21:3.

28. Holden S, Maksoud R, Eaton-Fitch N, Cabanas H, Staines D, MarshallGradisnik S. A systematic review of mitochondrial abnormalities in myalgic encephalomyelitis/chronic fatigue syndrome/systemic exertion intolerance disease. J Transl Med. 2020;18(1):290.

29. Missailidis D, Annesley SJ, Fisher PR. Pathological mechanisms underlying myalgic encephalomyelitis/chronic fatigue syndrome. Diagnostics. 2019;9:3.

30. Castro-Marrero J, Sáez-Francàs N, Segundo MJ, Calvo N, Faro M, Aliste L, et al. Effect of coenzyme Q10 plus nicotinamide adenine dinucleotide supplementation on maximum heart rate after exercise testing in chronic fatigue syndrome - A randomized, controlled, double-blind trial. Clin Nutr. 2016;35(4):826-34

31. Castro-Marrero J, Cordero MD, Segundo MJ, Sáez-Francàs N, Calvo N, Román-Malo L, et al. Does oral coenzyme Q10 plus NADH supplementation improve fatigue and biochemical parameters in chronic fatigue syndrome? Antioxid Redox Signal. 2015;22(8):679-85.

32. Forsyth LM, Preuss HG, MacDowell AL, Chiazze L, Birkmayer GD, Bellanti JA. Therapeutic effects of oral NADH on the symptoms of 
patients with chronic fatigue syndrome. Ann Allergy Asthma Immunol. 1999;82(2):185-91.

33. van Rosendal SP, Osborne MA, Fassett RG, Coombes JS. Guidelines for glycerol use in hyperhydration and rehydration associated with exercise. Sports Med. 2010;40(2):113-29.

34. Maher CG, Moseley AM, Sherrington C, Elkins MR, Herbert RD. A description of the trials, reviews, and practice guidelines indexed in the PEDro database. Phys Ther. 2008;88(9):1068-77.

35. Jadad AR, Moore RA, Carroll D, Jenkinson C, Reynolds DJ, Gavaghan DJ, et al. Assessing the quality of reports of randomized clinical trials: is blinding necessary? Control Clin Trials. 1996;17(1):1-12.

36. Verhagen AP, de Vet HC, de Bie RA, Kessels AG, Boers M, Bouter LM, et al. The Delphi list: a criteria list for quality assessment of randomized clinical trials for conducting systematic reviews developed by Delphi consensus. J Clin Epidemiol. 1998;51(12):1235-41.

37. Fukuda S, Nojima J, Kajimoto O, Yamaguti K, Nakatomi Y, Kuratsune $H$, et al. Ubiquinol-10 supplementation improves autonomic nervous function and cognitive function in chronic fatigue syndrome. BioFactors. 2016;42(4):431-40.

38. Ostojic SM, Stojanovic M, Drid P, Hoffman JR, Sekulic D, Zenic N. Supplementation with guanidinoacetic acid in women with chronic fatigue syndrome. Nutrients. 2016:8:2.

39. Montoya JG, Anderson JN, Adolphs DL, Bateman L, Klimas N, Levine SM, et al. KPAX002 as a treatment for myalgic encephalomyelitis/chronic fatique syndrome (ME/CFS): A prospective, randomized trial. Int J Clin Exp Med. 2018;11(3):2890-900.

40. Vermeulen RCW, Scholte HR. Exploratory Open Label, Randomized Study of Acetyl- and Propionylcarnitine in Chronic Fatigue Syndrome. Psychosom Med. 2004;66(2):276-82.

41. Menon R, Cribb L, Murphy J, Ashton MM, Oliver G, Dowling N, et al. Mitochondrial modifying nutrients in treating chronic fatigue syndrome: a 16-week open-label pilot study. Adv Integr Med. 2017;4(3):109-14.

42. Kaiser JD. A prospective, proof-of-concept investigation of KPAX002 in chronic fatigue syndrome. Int J Clin Exp Med. 2015;8(7):11064-74.
43. Nicolson GL. Mitochondrial dysfunction and chronic disease: treatment with natural supplements. Altern Ther Health Med. 2014;20(Suppl 1):18-25.

44. Comhaire F. Why do some ME/CFS patients benefit from treatment with sodium dichloroacetate, but others do not? Med Hypotheses. 2018;120:65-7.

45. Wyller VB. The chronic fatigue syndrome-an update. Acta Neurol Scand, Suppl. 2007;187:7-14

46. Silaidos C, Pilatus U, Grewal R, Matura S, Lienerth B, Pantel J, et al. Sexassociated differences in mitochondrial function in human peripheral blood mononuclear cells (PBMCs) and brain. Biol Sex Diff. 2018:9(1):34.

47. Allmark P. Should research samples reflect the diversity of the population? J Med Ethics. 2004;30(2):185-9.

48. Bushra R, Aslam N, Khan AY. Food-Drug Interactions. Oman Med J. 2011;26(2):77-83.

49. Althubaiti A. Information bias in health research: definition, pitfalls, and adjustment methods. J Multidiscip Healthc. 2016;4(9):211-7.

50. Rizzuto R, De Stefani D, Raffaello A, Mammucari C. Mitochondria as sensors and regulators of calcium signalling. Nat Rev Mol Cell Biol. 2012:13(9):566-78.

51. Wood E, Hall KH, Tate W. Role of mitochondria, oxidative stress and the response to antioxidants in myalgic encephalomyelitis/chronic fatigue syndrome: a possible approach to SARS-CoV-2'long-haulers'? Chronic Dis Transl Med. 2020.

52. Ostojic SM. Postviral fatigue syndrome and creatine: a piece of the puzzle? Nutr Neurosci. 2020;1:1-2.

\section{Publisher's Note}

Springer Nature remains neutral with regard to jurisdictional claims in published maps and institutional affiliations.
Ready to submit your research? Choose BMC and benefit from:

- fast, convenient online submission

- thorough peer review by experienced researchers in your field

- rapid publication on acceptance

- support for research data, including large and complex data types

- gold Open Access which fosters wider collaboration and increased citations

- maximum visibility for your research: over $100 \mathrm{M}$ website views per year

At BMC, research is always in progress.

Learn more biomedcentral.com/submissions 